\title{
Ready Conversion of Patients with Well-Controlled, Moderate to Severe, Chronic Malignant Tumor-related Pain on Other Opioids to Tapentadol Extended Release
}

\author{
Keiichiro Imanaka $\cdot$ Yushin Tominaga • \\ Mila Etropolski · Hiroki Ohashi · Keiichiro Hirose • \\ Taka Matsumura
}

Published online: 7 June 2014

(c) The Author(s) 2014. This article is published with open access at Springerlink.com

\begin{abstract}
Background and Objectives The effectiveness and tolerability of tapentadol extended release (ER), a centrally acting analgesic with $\mu$-opioid receptor agonist and norepinephrine (noradrenaline) reuptake inhibitor activities, have been demonstrated in patients with chronic pain, including those switching directly from prior opioid therapy. The objective of the current study was to evaluate the effectiveness and safety of conversion to oral tapentadol ER (50-250 mg twice daily) from previous around-theclock strong opioid therapy in patients with moderate to severe, chronic malignant tumor-related cancer pain that was well-controlled.

Methods This randomized, open-label, phase III study, which was conducted in Japan, included a 1- to 2-week screening period (on previous opioid) and an 8-week, openlabel treatment period. Eligible patients, who were taking a strong opioid analgesic and had a mean pain intensity score $<4$ during the 3 days prior to randomization (adequate
\end{abstract}

Previous presentations: The results of this study have previously been presented in part at the 18th Congress of the Japanese Society for Palliative Medicine, 21-22 June 2013, Yokohama, Japan.

ClinicalTrials.gov identifier: NCT01309386.

Electronic supplementary material The online version of this article (doi:10.1007/s40261-014-0204-3) contains supplementary material, which is available to authorized users.

K. Imanaka $(\bowtie) \cdot$ Y. Tominaga $\cdot$ H. Ohashi $\cdot$ K. Hirose $\cdot$

T. Matsumura

Janssen Japan, 5-2, Nishi-Kanda 3, Chiyoda-ku, Tokyo

101-0065, Japan

e-mail: kimanaka@its.jnj.com

M. Etropolski

Janssen Research \& Development, LLC, Raritan, NJ, USA pain control on previous strong opioid), were randomized (1:1) to receive twice-daily treatment with tapentadol ER (100-500 mg/day) or morphine sustained release (SR; 20-140 mg/day; reference for assay sensitivity). Initial doses were estimated based on the conversion ratio of tapentadol ER:oxycodone:morphine:fentanyl $=10: 2: 3: 0.03$. The primary effectiveness endpoint was the proportion of patients who maintained pain control [change from baseline in mean pain intensity (11-point numerical rating scale) less than +1.5 for 3 consecutive days and no more than two doses of rescue medication per day for 3 consecutive days) during the first week of open-label treatment.

Results In the tapentadol ER group $(n=50), 84.0 \%$ of patients $(42 / 50 ; 95 \%$ CI, 70.89-92.83) maintained pain control during Week 1. On the Patient Global Impression of Change, $2.1 \%$ (1/48), $2.1 \%$ (1/48), $22.9 \%$ (11/48), and $50.0 \%(24 / 48)$ of patients in the tapentadol ER group reported that their overall condition was "very much improved," "much improved," "minimally improved," and "not changed," respectively, at Week 1 compared with $0 \%, 10.7 \%$ (3/28), $28.6 \%$ (8/28), and $53.6 \%$ (15/28) reporting these ratings at Week 8 . The sensitivity of effectiveness analyses was validated based on results using morphine SR; $98.0 \%$ (49/50; $95 \%$ CI, 89.35-99.95) of patients in the morphine SR group maintained pain control after 1 week of treatment. The overall safety profile was similar to that demonstrated in previous studies; tapentadol ER was associated with a lower incidence of gastrointestinal treatment-emergent adverse events than morphine SR [38.0\% (19/50) vs. $54.0 \%(27 / 50)]$, including constipation $[12.0 \%(6 / 50)$ vs. $20.0 \%(10 / 50)]$ and vomiting [6.0\% (3/50) vs. $26.0 \%(13 / 50)]$.

Conclusions Overall, results indicate that conversion from previous strong opioids to tapentadol ER (50-250 mg twice daily) was successful and resulted in safe and 
effective pain control with improved gastrointestinal tolerability versus morphine SR in patients with moderate to severe cancer-related pain that was well-controlled on their previous opioid.

\section{Key Points}

Patients with moderate to severe cancer-related pain that was well-controlled on their previous opioid analgesic could be safely converted to tapentadol extended release (ER; 100-500 mg/day) with no loss of pain control.

The majority of patients maintained pain control within 1 week of conversion, indicating successful direct conversion from prior opioid therapy to tapentadol ER.

Tapentadol ER was associated with a better gastrointestinal tolerability profile than morphine sustained release $(20-140 \mathrm{mg} /$ day $)$.

\section{Introduction}

World Health Organization (WHO) Step III opioid analgesics (e.g., morphine) are often used for moderate to severe cancer pain management [1-3]. The level of pain relief achieved with opioid analgesics varies from patient to patient [1], and many patients (approximately 10-30\%) with cancer-related pain do not achieve adequate analgesia with opioids [4]. In addition, side effects are common in patients receiving long-term opioid treatment for chronic pain [1]. Gastrointestinal side effects (e.g., constipation, nausea, and vomiting) are particularly burdensome for patients with cancer-related pain [2, 4]. Opioids induce nausea, vomiting, and constipation in a high percentage of patients taking opioid analgesics for cancer pain management [5], and may exacerbate gastrointestinal symptoms associated with the underlying disease or treatment. Opioid switching is a proven approach for reducing intolerable side effects that has also been shown to be effective at improving analgesia for more than $50 \%$ of patients with a poor response to an initial opioid analgesic [6]. The Japanese Society of Palliative Medicine recommends switching the type of opioid used in patients with pain that is inadequately controlled or who experience intolerable side effects (particularly nausea and vomiting) when taking a particular opioid [7]. Improvements in pain intensity and tolerability have been observed following rotation from one opioid analgesic to another in multiple studies in Japanese patients with cancer pain [8-10].
Tapentadol is a centrally acting analgesic with two mechanisms of action: $\mu$-opioid receptor agonism and norepinephrine (noradrenaline) reuptake inhibition [11, 12]. The gastrointestinal tolerability of tapentadol extended release (ER) has been shown to be better than that of equianalgesic doses of oxycodone controlled release (CR) and morphine CR [13-17]. The effectiveness and safety of tapentadol ER for the management of moderate to severe, chronic tumor-related pain have been demonstrated in phase III clinical studies [16, 18] and in exploratory [19] and non-interventional [20] studies. In a phase III study in patients with moderate to severe, chronic tumor-related pain, tapentadol ER (100-250 mg twice daily) was shown to provide non-inferior effectiveness to that of morphine sulfate CR (40-100 mg twice daily) [16]. In a separate phase III study in opioid-naive Japanese patients experiencing cancer pain, the analgesic effectiveness of tapentadol ER (25-200 mg twice daily) was shown to be noninferior to that of oxycodone $\mathrm{HCl} \mathrm{CR} \mathrm{(5-40} \mathrm{mg} \mathrm{twice} \mathrm{daily)}$ [18]. Given its improved tolerability relative to opioid analgesics [13-16], tapentadol ER could be considered as a first option for patients who need opioid treatment to control their pain. For patients who are taking another strong opioid analgesic and experience intolerable opioid-induced side effects, it may be necessary to switch directly from the prior opioid to tapentadol ER to relieve those side effects.

In this 8-week, randomized, open-label phase III study (ClinicalTrials.gov identifier: NCT01309386) conducted in Japan, the effectiveness of tapentadol ER (50-250 mg twice daily) in safely converting patients from previous around-the-clock strong opioid therapy without loss of previous pain control was evaluated in patients with moderate to severe, chronic malignant tumor-related cancer pain that had been controlled by prior opioid therapy. Morphine sustained release (SR) was included as a reference for assay sensitivity in the current study, not as a direct comparator for effectiveness evaluations.

\section{Patients and Methods}

This study was conducted in accordance with the principles of the Declaration of Helsinki, Good Clinical Practice guidelines, and applicable regulatory requirements. The study protocol and amendments were reviewed and approved by Institutional Review Boards. Prior to study enrolment, all patients or their legally acceptable representatives provided their written consent to participate in the study after being provided with information regarding the nature and purpose of the study, participation/termination conditions, and the risks and benefits of treatment. This study was a registration trial in Japan and was 
requested by the Japanese Pharmaceuticals and Medical Devices Agency.

\subsection{Patient Population}

This study included men and women who were at least 20 years of age, had been diagnosed with cancer, and were receiving around-the-clock opioid therapy for moderate to severe, chronic malignant tumor-related cancer pain using one of the following: oral morphine SR $(\leq 120 \mathrm{mg} /$ day $)$, oral oxycodone $\mathrm{HCl} \mathrm{CR} \mathrm{(15-80} \mathrm{mg/day),} \mathrm{or} \mathrm{transdermal}$ fentanyl (Durotep ${ }^{\circledR}$ MT Patch, $\leq 8.4 \mathrm{mg} /$ patch; Fentos ${ }^{\circledR}$ Tape, $\leq 4 \mathrm{mg} /$ tape; OneDuro ${ }^{\circledR}$ Patch, $\leq 3.4 \mathrm{mg} /$ patch). During the 3 days prior to randomization, opioid doses must have been stable and the mean 24-h pain intensity score must have been $<4$ on an 11-point numerical rating scale (NRS) $[0=$ "no pain" to $10=$ "pain as bad as you can imagine"]. Eligible patients were allowed to use oral morphine immediate release (IR) or oxycodone IR as rescue medication for breakthrough pain. The dose per intake of rescue medication must have been no more than onesixth of the total daily dose (TDD) of the around-the-clock opioid analgesic, with conversion of the daily dose based on the ratio of morphine:oxycodone:fentanyl of 3:2:0.03; for patients who were taking morphine SR $(20 \mathrm{mg} /$ day $)$ or oxycodone $\mathrm{HCl} \mathrm{CR}(15 \mathrm{mg} /$ day $)$, the minimal strengths of rescue medication (morphine $\mathrm{HCl}$ IR $5 \mathrm{mg}$ or oxycodone $\mathrm{HCl}$ IR $2.5 \mathrm{mg}$ ) were used.

Exclusion criteria and prohibited and permitted medications are listed in the Electronic Supplementary Material.

\subsection{Study Design}

This randomized, open-label, parallel-arm, optimal dosetitration, multicenter study included a 1- to 2-week screening period and an 8-week open-label treatment period. During the study, patients were hospitalized or treated as outpatients. Patients continued taking the same doses of their previous around-the-clock daily opioid analgesics throughout the screening period. Eligible patients with a mean pain intensity score $<4$ during the 3 days prior to randomization were then randomized $(1: 1)$ to receive twice-daily treatment with tapentadol ER or morphine SR (as a reference for assay sensitivity) for 8 weeks. Randomization was stratified by prior opioid treatment to ensure that the treatment assignment was balanced. Morphine SR served as a reference for assay sensitivity, not as an active comparator, to validate the results observed with tapentadol ER. Starting doses of study drug were calculated based on the previous opioid analgesic dose (Table 1). Based on prior estimates of the equianalgesic ratio of tapentadol ER to oxycodone CR $(\sim 5: 1)[21,22]$ and of the equianalgesic ratio between oxycodone $\mathrm{CR}$, morphine $\mathrm{SR}$, and a fentanyl transdermal system (2:3:0.03) [23-26], conversion ratios between tapentadol ER and oxycodone, morphine, and fentanyl for the initial dose were estimated as tapentadol ER:oxycodone:morphine:fentanyl = 10:2:3: 0.03 . In patients switching from a fentanyl transdermal system, the system was to be removed at least $12 \mathrm{~h}$ before administration of the first dose of study drug. Patients who developed withdrawal symptoms after switching to study treatment could receive oral oxycodone IR or oral morphine IR (at an equivalent dose of one-fourth to one-tenth the TDD of the previously used opioid) for up to 3 days until symptoms disappeared. Patients were also permitted to take oral oxycodone IR or oral morphine IR as rescue medication for breakthrough pain throughout the study (including the screening and open-label treatment periods); the dose of rescue medication had to be no more than onesixth of the TDD of the around-the-clock opioid analgesics, including study drug. Patients with a daily dose of tapentadol ER $<100 \mathrm{mg}$ /day, morphine $\mathrm{SR}<20 \mathrm{mg}$ /day, or oxycodone $\mathrm{HCl} \mathrm{CR}<15 \mathrm{mg} /$ day were to use the minimal strengths of rescue medication (morphine $\mathrm{HCl}$ IR $5 \mathrm{mg}$ or oxycodone $\mathrm{HCl}$ IR $2.5 \mathrm{mg}$ ).

After at least 2 days of treatment with the initial dose, doses of study drug could be increased to a maximum of tapentadol ER $500 \mathrm{mg} /$ day or morphine SR $140 \mathrm{mg} / \mathrm{day}$ based on the investigator's judgment, which included evaluating if a patient's 24 -h NRS score was $\geq 4$ or had worsened compared with the previous day or if rescue medication for breakthrough pain was given $\geq 3$ times per day. For patients taking doses of tapentadol ER $<200 \mathrm{mg} /$ day and morphine SR $<60 \mathrm{mg} /$ day, doses could be increased in increments of tapentadol ER $50 \mathrm{mg} /$ day and morphine SR $10-20 \mathrm{mg} /$ day, respectively. For patients taking doses of tapentadol ER $\geq 200 \mathrm{mg} /$ day and morphine $\mathrm{SR} \geq 60 \mathrm{mg} /$ day, doses could be increased in increments of tapentadol ER $100 \mathrm{mg} /$ day or morphine SR $20-30 \mathrm{mg} / \mathrm{day}$, respectively. Doses could be reduced as needed during the study for safety reasons [e.g., adverse events (AEs)] to a minimum of tapentadol ER $50 \mathrm{mg} /$ day or morphine SR $20 \mathrm{mg} /$ day. Doses were titrated to each individual patient's optimal dose, balancing effectiveness and tolerability.

\subsection{Study Endpoints and Assessments}

Effectiveness in this study was evaluated as the ability to convert patients from previous, around-the-clock strong opioid therapy to tapentadol ER (50-250 mg twice daily) without loss of previous pain control. Patients rated their average pain intensity over the previous $24 \mathrm{~h}$ once daily on an 11-point NRS throughout the study. The primary effectiveness endpoint was defined as the proportion of patients who maintained pain control during the first week of the open-label treatment period. Pain control was 
Table 1 Starting daily dose of study drug based on dose of previous opioid treatment

\begin{tabular}{|c|c|c|c|c|c|c|}
\hline \multicolumn{5}{|c|}{ Previous opioid dose (mg) } & \multicolumn{2}{|c|}{ Starting dose (mg) } \\
\hline Oxycodone CR & Durotep $^{\circledR}$ MT Patch ${ }^{\mathrm{a}}$ & Fentos $^{\circledR}$ Tape $^{\mathrm{a}}$ & OneDuro ${ }^{\circledR}$ Patch $^{\mathrm{a}}$ & Morphine SR & Tapentadol ER & Morphine $\mathrm{SR}^{\mathrm{b}}$ \\
\hline$\geq 15$ to $\leq 20$ & 2.1 (0.3 mg/day) & 1 (0.3 mg/day) & 0.84 (0.3 mg/day) & $\geq 20$ to $\leq 30$ & 100 & 30 \\
\hline$>20$ to $\leq 30$ & - & - & - & $>30$ to $\leq 40$ & 150 & $40^{\mathrm{c}}$ \\
\hline$>30$ to $\leq 40$ & $4.2(0.6 \mathrm{mg} / \mathrm{day})$ & 2 (0.6 mg/day) & 1.7 (0.6 mg/day) & $>40$ to $\leq 60$ & 200 & 60 \\
\hline$>40$ to $\leq 60$ & $6.3(1.2 \mathrm{mg} /$ day $)$ & 3 (1.2 mg/day) & 2.54 (1.2 mg/day) & $>60$ to $\leq 90$ & 300 & 90 \\
\hline$>60$ to $\leq 80$ & 8.4 (1.8 mg/day) & 4 (1.8 mg/day) & 3.4 (1.8 mg/day) & $>90$ to $\leq 120$ & 400 & 120 \\
\hline
\end{tabular}

$C R$ controlled release, $E R$ extended release, $S R$ sustained release, - indicates absence of doses converted

${ }^{\text {a }}$ Previous opioid dose (mg) of Durotep ${ }^{\circledR}$ MT Patch, Fentos ${ }^{\circledR}$ Tape, and OneDuro ${ }^{\circledR}$ Patch indicates fentanyl content per patch or tape. Nominal release rate $(\mathrm{mg} / \mathrm{day})$ of fentanyl is shown in parentheses

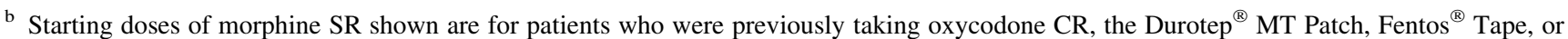
the OneDuro ${ }^{\circledR}$ Patch. Patients who were previously taking morphine SR continued on the same dose

c Although the dose calculated based on the equianalgesic ratio was $45 \mathrm{mg}$, a 40-mg dose was used because a 45-mg dose is not available

defined as having a change from baseline in the mean 24-h pain intensity score (11-point NRS) of less than +1.5 and using no more than two doses of rescue medication per day for any 3 consecutive days during the first week. Patients were enrolled in the study only if their pain was wellcontrolled [i.e., pain intensity (NRS) score $<4$ ] on their previous opioid therapy; thus, pain was considered to be controlled on study treatment only if patients experienced minimal changes in pain intensity after conversion from prior therapy.

Secondary effectiveness endpoints included average weekly pain intensity scores (11-point NRS), Patient Global Impression of Change (PGIC) ratings, and rescue medication use. The PGIC is a validated measure of global improvement with treatment for chronic pain [27, 28]. For the PGIC, patients completed the statement "Since the start of this treatment, my cancer-related pain overall is," using a 7-point scale $(1=$ "very much improved" to $7=$ "very much worse"). The PGIC was completed at Weeks 1, 2, 4, 6, and at the end of study or early withdrawal. The total number of days that patients took rescue medication, number of rescue medication doses taken in a calendar day, and TDD of rescue medication were evaluated for each patient.

Safety assessments included recorded AEs, clinical laboratory parameters, vital signs, 12-lead electrocardiograms (ECGs), and physical examinations. A treatmentemergent AE (TEAE) was defined as any AE that occurred on or after the first intake of study drug or that started before the first intake of study drug and worsened in intensity during the active treatment period. Incidences of TEAEs reported here include all TEAEs that occurred during the 8-week treatment period, and incidences of serious AEs reported here include all serious AEs that occurred during the 8-week treatment period and any spontaneously reported serious AEs within 7 days of the end of treatment.

\subsection{Statistical Analyses}

Based on results from previous studies with tapentadol and transdermal fentanyl [29], it was estimated that 49 patients should be included in the tapentadol ER group, assuming that the percentage of patients achieving pain control in this study was $85 \%$ and the lower limit of the 2-sided $95 \%$ confidence interval (CI) was $>75 \%(-10 \%)$. Morphine SR was used to evaluate assay sensitivity in this study, and the same number of patients were to be enrolled to receive morphine SR treatment as in the tapentadol ER group, bringing the total number of subjects to approximately 100 . The estimated number of patients to be enrolled by previous opioid treatment was as follows: morphine SR, $n=20$; oxycodone CR, $n=40$; fentanyl transdermal system, $n=40$.

In this study, the safety population included all randomized patients who received at least one dose of study medication. The full analysis population, which was used for all effectiveness analyses, included all randomized patients who received at least one dose of study medication and had post-baseline effectiveness data.

For baseline and demographic characteristics and study drug dosing data, descriptive statistics were used to summarize continuous variables, and frequency counts were used to summarize categorical variables. Study drug dosing information evaluated included the TDD of study drug, modal (or most frequently used) dose, duration of treatment, and the number of dose adjustments.

For the primary effectiveness analysis, the percentage (with $95 \% \mathrm{CI}$ ) of patients who maintained pain control during the first week of treatment was calculated. Patients who discontinued after $<3$ days of treatment or who did not have any available 24-h pain intensity scores (11-point NRS) for 3 consecutive days during the first week of treatment were considered to have not maintained pain 
control. The proportion of patients who maintained pain control was also evaluated by previous opioid treatment, sex, and age group $(<65$ and $\geq 65$ years of age).

The average weekly pain intensity score (11-point NRS), change from baseline in average pain intensity, and percentage change from baseline in pain intensity were summarized using descriptive statistics. PGIC ratings were summarized using frequency counts.

The number of doses of rescue medication taken, the TDD of rescue medication (converted into morphine equivalent doses using the conversion rate of dosage from oxycodone to morphine of 1.5), and total number of days with rescue medication during each week of the treatment period and during the overall treatment period were summarized using descriptive statistics.

Frequency counts were used to summarize the incidence of TEAEs, deaths and serious AEs, and AEs leading to treatment discontinuation. The incidence of TEAEs was also analyzed by previous opioid treatment, sex, and age group ( $<65$ and $\geq 65$ years of age).

In the current study, morphine SR served as a reference for assay sensitivity, not as an active comparator, so no formal comparisons of the effectiveness results for tapentadol ER and morphine SR were performed. For that reason, effectiveness data for the patients in the tapentadol
ER and morphine SR groups are presented separately; sensitivity analyses (i.e., effectiveness results for morphine $\mathrm{SR})$ are included in the Electronic Supplementary Material. Patient disposition and demographic data are presented together to show that the population analyzed for effectiveness (the tapentadol ER group) was comparable with the population analyzed for assay sensitivity (the morphine SR group). Safety and tolerability data for both treatment groups are presented together to allow for informal comparisons of the tolerability of tapentadol ER with that of an opioid analgesic commonly used for cancer pain management.

\section{Results}

\subsection{Patients}

This study was conducted from 23 August 2010 to 17 January 2012. Overall, 100 patients were randomized at 27 sites in Japan and took at least one dose of study drug (Fig. 1); enrolment was evenly distributed across study sites. In both the tapentadol ER group and the morphine SR (reference for assay sensitivity) group, $>55 \%$ of patients completed the treatment period; the most common reasons
Fig. 1 Patient disposition. $E R$ extended release, $S R$ sustained release

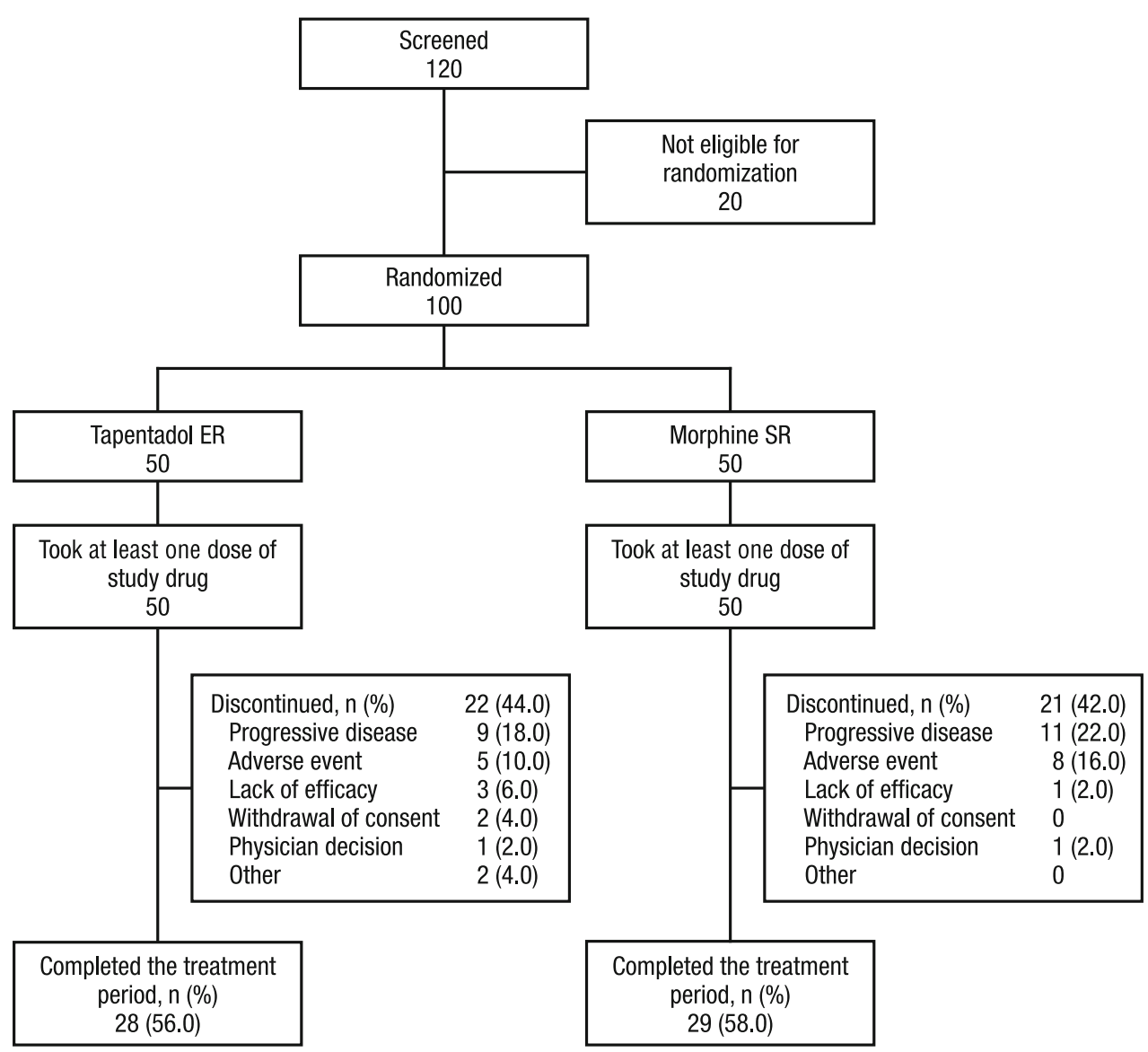


for discontinuation were progressive disease (reflecting the severity of their primary diagnosis of cancer) and AEs (Fig. 1).

In the tapentadol ER group, $26.0 \%(13 / 50)$ of patients had previously taken morphine SR, $44.0 \%$ (22/50) had previously taken oxycodone CR, and $30.0 \%(15 / 50)$ had previously taken transdermal fentanyl. In the morphine SR (reference for assay sensitivity) group, $24 \%$ (12/50) of patients had previously taken morphine SR, $46 \%(23 / 50)$ of patients had previously taken oxycodone CR, and $30 \%$ $(15 / 50)$ of patients had previously taken transdermal fentanyl. Baseline and demographic characteristics for the tapentadol ER group and the morphine SR (reference for assay sensitivity) group are summarized in Table 2.

\subsection{Tapentadol Extended Release Treatment Exposure}

In the tapentadol ER group, the median duration of treatment during the 8-week treatment period was 54.5 days. The majority $[70.0 \%(35 / 50)]$ of patients in the tapentadol ER group received study treatment for $>28$ to $\leq 56$ days. The mean (standard deviation [SD]) average TDD of tapentadol ER was 147.2 (91.06) $\mathrm{mg}$ during the first week of study treatment; over the entire 8-week treatment period, the mean (SD) average TDD of tapentadol ER was 173.5 (101.51) mg. The median modal TDD of tapentadol was $100.0 \mathrm{mg}$ during the first week of treatment and $150.0 \mathrm{mg}$ over the entire 8-week treatment period. Patients in the tapentadol ER group took the modal dose for a median of 7.0 days during the first week and a median of 38.0 days over the entire 8-week treatment period. In the tapentadol ER group, $28.0 \%(14 / 50)$ of patients had a dose increase during the first week of treatment, and $58.0 \%$ (29/50) of patients had a dose increase over the entire 8-week treatment period (Table 3).

Table 2 Baseline and demographic characteristics (safety population)

\begin{tabular}{lcr}
\hline Characteristic & $\begin{array}{l}\text { Tapentadol ER } \\
(n=50)\end{array}$ & $\begin{array}{l}\text { Morphine SR } \\
(n=50)\end{array}$ \\
\hline Age, years & $66.4(8.54)$ & $63.8(9.97)$ \\
Age category, $n(\%)$ & $23(46.0)$ & $25(50.0)$ \\
$\quad<65$ years & $27(54.0)$ & $25(50.0)$ \\
$\geq 65$ years & & \\
Sex, $n(\%)$ & $25(50.0)$ & $27(54.0)$ \\
Male & $25(50.0)$ & $23(46.0)$ \\
Female & $19.7(3.11)$ & $20.3(3.31)$ \\
BMI $\left(\mathrm{kg} / \mathrm{m}^{2}\right)$ & $1.5(1.11)$ & $1.8(1.14)$ \\
Baseline pain intensity score &
\end{tabular}

Values are expressed as mean (standard deviation) unless otherwise specified

$B M I$ body mass index, $E R$ extended release, $S R$ sustained release
Table 3 Dose adjustments in the tapentadol extended release group during the 8-week treatment period ${ }^{\mathrm{a}}$

\begin{tabular}{lc}
\hline $\begin{array}{l}\text { Change category } \\
\text { Number of changes }\end{array}$ & $\begin{array}{l}\text { Tapentadol ER } \\
(n=50)\end{array}$ \\
\hline Dose decreases & $44(88.0)$ \\
1 & $32(72.7)$ \\
2 & $6(13.6)$ \\
3 & $5(11.4)$ \\
$>3$ & $1(2.3)$ \\
No change & $5(10.0)$ \\
Dose increases & $29(58.0)$ \\
1 & $6(20.7)$ \\
2 & $13(44.8)$ \\
3 & $2(6.9)$ \\
$>3$ & $8(27.6)$ \\
\hline
\end{tabular}

Values are expressed as $n(\%)$

$E R$ extended release

${ }^{a}$ Missed doses were not considered dose changes

b Percentages were calculated out of the total number of patients

${ }^{c}$ Percentages were calculated out of the number of patients with dose decreases or increases

\subsection{Effectiveness}

For the primary effectiveness endpoint, the percentages of patients taking tapentadol ER who maintained pain control (with $95 \%$ CIs) during the first week of treatment overall and by prior opioid treatment are summarized in Table 4 . Of 50 patients taking tapentadol ER, only eight did not maintain pain control; two of those patients did not have pain intensity scores for 3 consecutive days, two failed to meet the pain intensity criterion, two failed to meet the rescue medication criterion, and two failed to meet both the pain intensity and rescue medication criteria. Similar percentages of men $[80.0 \%(20 / 25)]$ and women $[88.0 \%$ (22/ 25)] who were receiving tapentadol ER maintained pain control during the first week of treatment; the percentage of patients who maintained pain control was also similar across the two age groups evaluated $[<65$ years, $82.6 \%$ (19/23); $\geq 65$ years, $85.2 \%$ (23/27)].

The mean (SD) baseline pain intensity score (on prior opioid treatment; averaged over the 3 days prior to randomization) for patients in the tapentadol ER group was 1.5 (1.11). After switching from previous opioid analgesics to tapentadol ER, mean average weekly pain intensity scores were $\leq 2$ during the entire 8 -week treatment period. Mean changes from baseline in average pain intensity were $\leq 0.4$ throughout the treatment period. The mean average weekly pain intensity score increased to slightly above the baseline value at Week 1 (mean [SD] change from baseline to Week 1, 0.4 [0.92]) but thereafter returned progressively 
Table 4 Percentage of patients taking tapentadol extended release who maintained pain control overall and by prior treatment (full analysis population)

\begin{tabular}{|c|c|c|c|c|}
\hline & \multirow{2}{*}{$\begin{array}{l}\text { Total } \\
(n=50)\end{array}$} & \multicolumn{3}{|c|}{ Previous treatment } \\
\hline & & $\begin{array}{l}\text { Morphine SR } \\
(n=13)\end{array}$ & $\begin{array}{l}\text { Oxycodone CR } \\
(n=22)\end{array}$ & $\begin{array}{l}\text { Transdermal fentanyl } \\
(n=15)\end{array}$ \\
\hline Patients achieving pain control, $n(\%)$ & $42(84.0)$ & $9(69.2)$ & $20(90.9)$ & $13(86.7)$ \\
\hline $95 \% \mathrm{CI}$ & $70.89-92.83$ & $38.57-90.91$ & $70.84-98.88$ & $59.54-98.34$ \\
\hline
\end{tabular}

$C I$ confidence interval, $C R$ controlled release, $E R$ extended release, $S R$ sustained release

to the baseline value by Week 8 (mean [SD] change from baseline to Week 8, $0.0[0.92])$.

PGIC scores at Weeks 1,4 , and 8 are summarized in Fig. 2. None of the patients taking tapentadol ER reported a rating of "very much worse" at any timepoint. It is noteworthy that, although patients experienced adequate pain control on their previous opioid analgesic (a criterion for inclusion in the study), there were still some patients who reported improvements in their overall condition following conversion to tapentadol ER (Fig. 2).

Patients in the tapentadol ER group took rescue medication for a mean (SD) of 15.9 (19.58) days during the 8-week treatment period. At baseline (on prior opioid treatment), the mean (SD) number of doses of rescue medication that
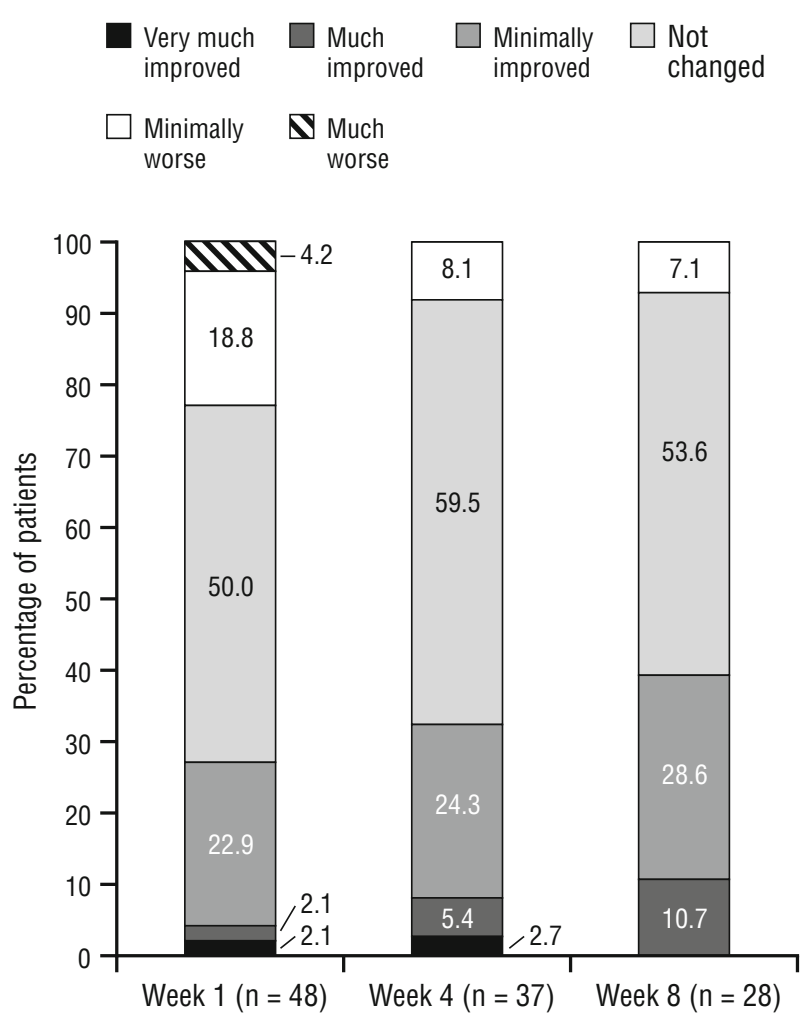

Fig. 2 Patient Global Impression of Change ratings at Weeks 1, 4, and 8 for patients taking tapentadol extended release (full analysis population). None of the patients reported a rating of "very much worse" at Week 1,4 , or 8 patients in the tapentadol ER group took per day was 0.4 (0.62). The mean (SD) number of doses of rescue medication taken per day by patients in the tapentadol ER group was similar to baseline during Week 1 [0.7 (0.99)], Week 4 [0.7 (0.96)], and Week 8 [0.5 (0.76)]. The mean (SD) average TDD (converted into morphine equivalent doses) of rescue medication used by patients in the tapentadol ER group decreased over the course of the treatment period, from 2.9 (8.34) mg during Week 1 to 1.8 (8.27) mg during Week 8; for the overall treatment period, the mean (SD) average TDD of rescue medication was $3.0(8.30) \mathrm{mg}$.

\subsection{Safety and Tolerability}

Overall, $90.0 \%(45 / 50)$ of patients in the tapentadol ER group and $94.0 \%$ (47/50) of patients in the morphine SR (reference for assay sensitivity) group reported at least one TEAE during the 8 -week treatment period. The most common TEAEs (incidence $\geq 5 \%$ in either treatment group) are summarized in Table 5. Disease progression was the most common TEAE in both treatment groups, which reflects the disease state of cancer patients who need opioid analgesics for their pain. The incidence of gastrointestinal TEAEs during the 8-week treatment period was numerically lower in the tapentadol ER group than in the morphine SR group, particularly the TEAEs of constipation and vomiting. During Week 1, $42.0 \%$ (21/50) of patients in the tapentadol ER group and $60.0 \%$ (30/50) of patients in the morphine SR group reported at least one TEAE. Gastrointestinal TEAEs were among the most common TEAEs reported during Week 1; in the tapentadol ER and morphine SR groups, respectively, $4.0 \%(2 / 50)$ and $14.0 \%(7 / 50)$ of patients reported nausea, $2.0 \%(1 / 50)$ and $16.0 \%(8 / 50)$ reported vomiting, and $2.0 \%(1 / 50)$ and $14.0 \%(7 / 50)$ reported constipation.

In the tapentadol ER group, at least one TEAE was reported during the 8 -week treatment period by $84.6 \%$ (11/13) of patients who previously took morphine SR, $95.5 \%(21 / 22)$ of patients who previously took oxycodone CR, and $86.7 \%(13 / 15)$ of patients who previously took transdermal fentanyl. In the morphine SR group, at least one TEAE was reported during the treatment period by 
Table 5 Incidence of treatment-emergent adverse events reported by $\geq 5 \%$ of patients in either treatment group (safety population)

\begin{tabular}{|c|c|c|}
\hline $\begin{array}{l}\text { System organ class } \\
\text { TEAE }\end{array}$ & $\begin{array}{l}\text { Tapentadol } \\
\text { ER } \\
(n=50)\end{array}$ & $\begin{array}{l}\text { Morphine } \\
\text { SR } \\
(n=50)\end{array}$ \\
\hline $\begin{array}{l}\text { General disorders and administration-site } \\
\text { conditions }\end{array}$ & $21(42.0)$ & $20(40.0)$ \\
\hline Disease progression & $16(32.0)$ & $17(34.0)$ \\
\hline Malaise & $4(8.0)$ & 0 \\
\hline Pyrexia & $3(6.0)$ & $4(8.0)$ \\
\hline Nervous system disorders & $18(36.0)$ & $17(34.0)$ \\
\hline Somnolence & $8(16.0)$ & $10(20.0)$ \\
\hline Headache & $1(2.0)$ & $4(8.0)$ \\
\hline Gastrointestinal disorders & $19(38.0)$ & $27(54.0)$ \\
\hline Nausea & $7(14.0)$ & $7(14.0)$ \\
\hline Constipation & $6(12.0)$ & $10(20.0)$ \\
\hline Vomiting & $3(6.0)$ & $13(26.0)$ \\
\hline Diarrhea & $3(6.0)$ & $4(8.0)$ \\
\hline Blood and lymphatic system disorders & $8(16.0)$ & $3(6.0)$ \\
\hline Anemia & $4(8.0)$ & $1(2.0)$ \\
\hline Psychiatric disorders & $5(10.0)$ & $1(2.0)$ \\
\hline Insomnia & $4(8.0)$ & 0 \\
\hline $\begin{array}{l}\text { Injury, poisoning, and procedural } \\
\text { complications }\end{array}$ & $4(8.0)$ & $4(8.0)$ \\
\hline Fall & $4(8.0)$ & $1(2.0)$ \\
\hline Investigations & $8(16.0)$ & $13(26.0)$ \\
\hline$\gamma$-Glutamyltransferase increased & $1(2.0)$ & $4(8.0)$ \\
\hline Skin and subcutaneous tissue disorders & $8(16.0)$ & $10(20.0)$ \\
\hline Pruritus & $1(2.0)$ & $4(8.0)$ \\
\hline
\end{tabular}

Values are expressed as $n(\%)$

$E R$ extended release, $S R$ sustained release, $T E A E$ treatment-emergent adverse event

$75.0 \%(9 / 12)$ of patients who previously took morphine SR, $100.0 \%(23 / 23)$ of patients who previously took oxycodone $\mathrm{CR}$, and $100.0 \%(15 / 15)$ of patients who previously took transdermal fentanyl. The overall incidence of TEAEs was similar for male and female patients in the tapentadol ER group $[92.0 \%(23 / 25)$ vs. $88.0 \%(22 / 25)]$ and in the morphine SR group [96.3\% (26/27) vs. $91.3 \%$ (21/23)]. For patients $<65$ and $\geq 65$ years of age, respectively, the overall incidences of TEAEs were $95.7 \%$ (22/23) and $85.2 \%$ (23/ $27)$ in the tapentadol ER group and $96.0 \%(24 / 25)$ and $92.0 \%(23 / 25)$ in the morphine SR group.

Drug-related TEAEs were less common with tapentadol ER [38.0\% (19/50)] than with morphine SR [64.0\% $(32 / 50)]$, as were TEAEs leading to treatment discontinuation $[28.0 \%(14 / 50)$ vs. $38.0 \%(19 / 50)]$. The most common TEAEs leading to treatment discontinuation (incidence $>2 \%$ in either treatment group) were disease progression [tapentadol ER, $18.0 \%$ (9/50); morphine SR,
$22.0 \%(11 / 50)]$ and vomiting [tapentadol ER, 0; morphine SR, $8.0 \%(4 / 50)]$.

Overall, $32.0 \%(16 / 50)$ of patients in the tapentadol ER group and $32.0 \%(16 / 50)$ of patients in the morphine SR group had a serious TEAE. There were six deaths in the tapentadol ER group; five of these deaths were due to disease progression and were considered by the investigator to be unrelated to study drug, and one was due to gastrointestinal perforation that occurred 30 days after starting tapentadol ER and was considered by the investigator to be doubtfully related to study drug. There were four deaths in the morphine SR group, all of which were due to disease progression and were considered by the investigator to be unrelated to study drug.

In the tapentadol ER group, there were no unexpected safety results attributable to treatment based on laboratory, vital sign, and ECG values.

\section{Discussion}

Results of this study showed that conversion from opioid analgesics (morphine SR, oxycodone CR, or transdermal fentanyl) to tapentadol ER was successful in patients with moderate to severe, chronic malignant tumor-related pain that was well-controlled on their previous opioid; pain control was maintained after switching to tapentadol ER treatment and tapentadol ER was generally well-tolerated. This study design did not follow the switching criteria typically used in clinical practice, which generally involves switching opioid analgesics only for patients who experience inadequate pain control or intolerable side effects on their current opioid. The intention of the current study was to show under controlled experimental conditions that patients with stable pain control could achieve comparable effectiveness with tapentadol ER, in order to be able to recommend this switch as necessary in practice. Pain control was maintained by $84 \%$ of patients within the first week after switching to tapentadol ER. For the primary effectiveness endpoint, a 7-day period after switching from the previous strong opioid analgesic was considered sufficient to demonstrate pain control because the initial dose of study drug was based on the dose of the previous opioid, which had provided adequate pain control [as indicated by a pain intensity (NRS) score <4]. Although pain control was consistent following direct conversion from prior opioid therapy to tapentadol ER, effectiveness in the tapentadol ER group further improved over time after Week 1; this further improvement indicates that the best effectiveness was obtained after the initial conversion when patients were individually titrated to their optimal dose, balancing effectiveness and tolerability. The sensitivity of effectiveness analyses in the current study were 
validated based on the positive results achieved with morphine SR; a high percentage of patients (98\%) maintained pain control during the first week with morphine SR. Morphine SR was chosen as the reference for assay sensitivity in this study because it is generally considered to be the standard of care for the management of moderate to severe cancer pain requiring a strong opioid and has been used extensively in cancer pain studies [30]. Because this study included patients who were previously on strong opioid analgesics (including morphine SR), some patients who were previously taking morphine SR would have been randomized to the same treatment (morphine SR) during openlabel treatment, which may have introduced bias in favor of the morphine SR group; in fact, $24 \%$ of patients in the morphine SR group were pretreated with morphine SR prior to conversion. For that reason, direct comparisons of effectiveness between the tapentadol ER group, in which all patients had previously received other strong opioids (morphine SR, oxycodone CR, or transdermal fentanyl), and the morphine SR treatment group may not have yielded accurate results for interpretation of conversion in this group.

PGIC outcomes supported the results of the primary effectiveness analysis in the tapentadol ER group; the percentage of patients reporting an improvement or no change in their overall condition on the PGIC increased over the course of the 8-week treatment period. Only patients with adequate pain control on their previous opioid analgesic were included in this study, so major improvements were not expected in patients' overall condition. With tapentadol ER, the majority of patients experienced no change or a minimal improvement; this is consistent with the inclusion of a population with well-controlled pain on their previous analgesic regimen, but also suggests that the majority of patients treated with tapentadol ER either maintained or experienced improvements in their pain control and overall condition.

In the current study, tapentadol ER was generally welltolerated and there were no unexpected safety findings. The incidence of gastrointestinal TEAEs (particularly vomiting and constipation), drug-related AEs, and AEs leading to discontinuation was lower with tapentadol ER than with morphine SR. Additionally, the differences observed in this study between tapentadol ER and morphine SR in gastrointestinal tolerability and in AEs leading to discontinuation were comparable with differences seen between tapentadol and oxycodone in other phase III studies in acute and chronic pain [13, 14, 31-33]. The improvement in gastrointestinal side effects with tapentadol ER may be of particular clinical relevance because gastrointestinal side effects often lead patients to discontinue therapy, resulting in disruption of pain relief $[34,35]$.

The conversion ratio used in the current study to determine starting doses of tapentadol ER and morphine SR was based on prior estimates of the equianalgesic ratio of tapentadol ER to oxycodone CR $(\sim 5: 1)[21,22]$ and of the equianalgesic ratio between oxycodone $\mathrm{CR}$, morphine $\mathrm{SR}$, and a fentanyl transdermal system (2:3:0.03) [23-26]. The tapentadol ER to morphine SR conversion ratio in the current study (3.3:1) was in keeping with previously reported estimates $[17,21]$. There is generally a range of estimates of dose conversion ratios for opioid analgesics $[6,36]$, and the conversion ratios used in the current study were at the lower end of those ranges. This type of conservative approach may be associated with a better response to opioid treatment and a more favorable tolerability profile than a more aggressive treatment strategy $[6,36]$.

The daily doses of tapentadol ER used in the current study were relatively low. For tapentadol ER, the mean average TDD used in the current study was $173.5 \mathrm{mg}$; by comparison, the mean average TDD of tapentadol ER used in previous studies in patients with chronic osteoarthritis or low back pain ranged from 232.7 to $326.7 \mathrm{mg}$ [13, 14, 21, 22, 31, 37]. The difference in TDDs between the current study and those previous studies may have been related to differences in study populations. The previous studies included largely North American and European patients [13, 14, 21, 22, 31, 37], while the current study included exclusively Japanese patients. In general, Japanese patients require lower doses of opioid-type analgesics to control their cancer pain than Western patients [18, 38-40].

This study may be associated with some inherent limitations, including the fact that the study is not double-blind. The open-label design of this first conversion study with tapentadol ER in Japanese patients was intended to ensure the safety of study participants and to allow proper dosing decisions to be made for these patients. In addition, some patients randomized to treatment in the morphine SR group had previously been taking morphine SR; results for those patients, who received continuous treatment with a single opioid may have differed from results for patients who switched from a different previous opioid to morphine SR or tapentadol ER. The positive results achieved in the current study, which showed that tapentadol ER was safe and effective for chronic cancer pain management after direct conversion from WHO Step III opioids, were congruous with those of previous studies showing safe and effective pain relief with tapentadol ER after direct conversion from WHO Step III opioids in patients with different types of chronic pain [21, 22].

Overall, results of this study indicate that patients whose moderate to severe, cancer-related pain was previously well-controlled with morphine SR, oxycodone $\mathrm{CR}$, or transdermal fentanyl were safely converted to tapentadol ER (50-250 mg twice daily) with no loss of pain control. The majority of patients had maintained pain control within 1 week of conversion to tapentadol ER, indicating that direct 
conversion from prior strong opioid therapy to tapentadol ER was successful. In addition, tapentadol ER had a better gastrointestinal tolerability profile than morphine SR; a notable difference in the incidence of gastrointestinal AEs between tapentadol ER and morphine SR was observed after only 1 week of treatment. The favorable tolerability profile of tapentadol ER may improve compliance in patients with chronic cancer-related pain, resulting in more consistent pain relief and better patient outcomes.

Acknowledgments Janssen Research \& Development, LLC, funded this study. Editorial support for the writing of this manuscript was provided by Megan Knagge, $\mathrm{PhD}$, of MedErgy, and was funded by Janssen Research \& Development, LLC. The authors retained full editorial control over the content of the manuscript.

Conflict of interest $\mathrm{KI}$ is an employee of Janssen Japan and a Johnson \& Johnson stockholder. YT, HO, KH, and TM are employees of Janssen Japan. ME is an employee of Janssen Research \& Development, LLC, and a Johnson \& Johnson stockholder.

Open Access This article is distributed under the terms of the Creative Commons Attribution Noncommercial License which permits any noncommercial use, distribution, and reproduction in any medium, provided the original author(s) and the source are credited.

\section{References}

1. Droney J, Riley J. Recent advances in the use of opioids for cancer pain. J Pain Res. 2009;2:135-55.

2. Mandala M, Moro C, Labianca R, et al. Optimizing use of opiates in the management of cancer pain. Ther Clin Risk Manag. 2006;2:447-53.

3. Mercadante S. Emerging drugs for cancer-related pain. Support Care Cancer. 2011;19:1887-93.

4. Cherny N, Ripamonti C, Pereira J, et al. Strategies to manage the adverse effects of oral morphine: an evidence-based report. J Clin Oncol. 2001;19:2542-54.

5. Caraceni A, Hanks G, Kaasa S, et al. Use of opioid analgesics in the treatment of cancer pain: evidence-based recommendations from the EAPC. Lancet Oncol. 2012;13:e58-68.

6. Mercadante S, Bruera E. Opioid switching: a systematic and critical review. Cancer Treat Rev. 2006;32:304-15.

7. Yamaguchi T, Shima Y, Morita T, et al. Clinical guideline for pharmacological management of cancer pain: the Japanese Society of Palliative Medicine recommendations. Jpn J Clin Oncol. 2013;43:896-909.

8. Narabayashi M, Saijo Y, Takenoshita S, et al. Opioid rotation from oral morphine to oral oxycodone in cancer patients with intolerable adverse effects: an open-label trial. Jpn J Clin Oncol. 2008;38:296-304.

9. Takakuwa O, Oguri T, Maeno K, et al. Analgesic effect of switching from oral opioids to a once-a-day fentanyl citrate transdermal patch in patients with lung cancer. Am J Hosp Palliat Care. 2013;30:726-9.

10. Tani M, Fuku A, Terashita S, et al. Early conversion from oral morphine to transdermal fentanyl in patients with cancer pain. Hepatogastroenterology. 2008;55:1545-8.

11. Tzschentke TM, De Vry J, Terlinden R, et al. Tapentadol hydrochloride. Analgesic, mu-opioid receptor agonist, noradrenaline reuptake inhibitor. Drugs Future. 2006;31:1053-61.
12. Tzschentke TM, Christoph T, Kögel B, et al. (-)-(1R,2R)-3-(3Dimethylamino-1-ethyl-2-methyl-propyl)-phenol hydrochloride (tapentadol $\mathrm{HCl}$ ): a novel $\mu$-opioid receptor agonist/norepinephrine reuptake inhibitor with broad-spectrum analgesic properties. J Pharmacol Exp Ther. 2007;323:265-76.

13. Afilalo M, Etropolski MS, Kuperwasser B, et al. Efficacy and safety of tapentadol extended release compared with oxycodone controlled release for the management of moderate to severe chronic pain related to osteoarthritis of the knee: a randomized, double-blind, placebo- and active-controlled phase III study. Clin Drug Investig. 2010;30:489-505.

14. Buynak R, Shapiro DY, Okamoto A, et al. Efficacy and safety of tapentadol extended release for the management of chronic low back pain: results of a prospective, randomized, double-blind, placebo- and active-controlled phase III study. Expert Opin Pharmacother. 2010;11:1787-804.

15. Lange B, Kuperwasser B, Okamoto A, et al. Efficacy and safety of tapentadol prolonged release for chronic osteoarthritis pain and low back pain. Adv Ther. 2010;27:381-99.

16. Kress HG, Koch ED, Hosturski H, et al. Efficacy and safety of oral tapentadol extended release (ER) for the management of moderate to severe, chronic malignant tumor-related pain [abstract no. A20]. American Society of Regional Anesthesia and Pain Medicine (ASRA) 11th Annual Pain Medicine Meeting; 12-15 Nov 2012; Miami.

17. Mercadante S, Porzio G, Aielli F, et al. Opioid switching from and to tapentadol extended release in cancer patients: conversion ratio with other opioids. Curr Med Res Opin. 2013;29:661-6.

18. Imanaka K, Tominaga Y, Etropolski M, et al. Efficacy and safety of oral tapentadol extended release in Japanese and Korean patients with moderate to severe, chronic malignant tumor-related pain. Curr Med Res Opin. 2013;29:1399-409.

19. Mercadante S, Porzio G, Ferrera P, et al. Tapentadol in cancer pain management: a prospective open-label study. Curr Med Res Opin. 2012;28:1775-9.

20. Schwenke KM, Litzenburger BC. Tapentadol PR in the treatment of cancer pain in clinical practice: first data [poster]. The International Association for the Study of Pain (IASP) 14th World Congress on Pain; 27-31 Aug 2012; Milan.

21. Gálvez R, Schäfer M, Hans G, et al. Tapentadol prolonged release versus strong opioids for severe, chronic low back pain: results of an open-label, phase IIIb study. Adv Ther. 2013;30:229-59.

22. Steigerwald I, Schenk M, Lahne U, et al. Effectiveness and tolerability of tapentadol prolonged release compared with prior opioid therapy for the management of severe, chronic osteoarthritis pain. Clin Drug Investig. 2013;33:607-19.

23. Donner B, Zenz M, Tryba M, et al. Direct conversion from oral morphine to transdermal fentanyl: a multicenter study in patients with cancer pain. Pain. 1996;64:527-34.

24. OXYCONTIN ${ }^{\circledR}$ (oxycodone hydrochloride controlled-release) tablets [package insert]. Stamford: Purdue Pharma L.P.; 2010.

25. Fumikazu T, Hideo Y, Yasuo K, et al. Clinical evaluation of cancer patients who switched from controlled-release morphine sulfate tablets to S-8117 (controlled release oxycodone hydrochloride tablets)—phase II clinical study. J Clin Ther Med. 2005;21:281-93.

26. Japanese Society for Palliative Medicine. Clinical guidelines for cancer pain management. Tokyo: Kanehara and Co., Ltd; 2005.

27. Dworkin RH, Turk DC, Farrar JT, et al. Core outcome measures for chronic pain clinical trials: IMMPACT recommendations. Pain. 2005;113:9-19.

28. Farrar JT, Young JP Jr, LaMoreaux L, et al. Clinical importance of changes in chronic pain intensity measured on an 11-point numerical pain rating scale. Pain. 2001;94:149-58. 
29. Hanaoka K, Yoshimura T, Tomioka T, et al. Clinical study of one-day fentanyl patch in patients with cancer pain-evaluation of the efficacy and safety in relation to treatment switch from opioid analgesic therapy. Jpn J Anesthesiol (Masui). 2011;60:147-56.

30. Wiffen PJ, McQuay HJ. Oral morphine for cancer pain. Cochrane Database Syst Rev. 2007;(4):CD003868.

31. Wild JE, Grond S, Kuperwasser B, et al. Long-term safety and tolerability of tapentadol extended release for the management of chronic low back pain or osteoarthritis pain. Pain Pract. 2010;10:416-27.

32. Daniels S, Casson E, Stegmann JU, et al. A randomized, doubleblind, placebo-controlled phase 3 study of the relative efficacy and tolerability of tapentadol IR and oxycodone IR for acute pain. Curr Med Res Opin. 2009;25:1551-61.

33. Hartrick C, Van Hove I, Stegmann J-U, et al. Efficacy and tolerability of tapentadol immediate release and oxycodone $\mathrm{HCl}$ immediate release in patients awaiting primary joint replacement surgery for end-stage joint disease: a 10-day, phase III, randomized, double-blind, active- and placebo-controlled study. Clin Ther. 2009;31:260-71.

34. Panchal SJ, Muller-Schwefe P, Wurzelmann JI. Opioid-induced bowel dysfunction: prevalence, pathophysiology and burden. Int $\mathbf{J}$ Clin Pract. 2007;61:1181-7.
35. Candrilli SD, Davis KL, Iyer S. Impact of constipation on opioid use patterns, health care resource utilization, and costs in cancer patients on opioid therapy. J Pain Palliat Care Pharmacother. 2009;23:231-41.

36. Mercadante S. Opioid rotation for cancer pain: rationale and clinical aspects. Cancer. 1999;86:1856-66.

37. Steigerwald I, Muller M, Davies A, et al. Effectiveness and safety of tapentadol prolonged release for severe, chronic low back pain with or without a neuropathic pain component: results of an openlabel, phase 3b study. Curr Med Res Opin. 2012;28:911-36.

38. Kosugi T, Hamada S, Takigawa $\mathrm{C}$, et al. A randomized, doubleblind, placebo-controlled study of fentanyl buccal tablets for breakthrough pain: efficacy and safety in japanese cancer patients. J Pain Symptom Manage. Epub 2013 Oct 5.

39. Koizumi W, Toma H, Watanabe K, et al. Efficacy and tolerability of cancer pain management with controlled-release oxycodone tablets in opioid-naive cancer pain patients, starting with $5 \mathrm{mg}$ tablets. Jpn J Clin Oncol. 2004;34:608-14.

40. Duthey B, Scholten W. Adequacy of opioid analgesic consumption at country, global, and regional levels in 2010, its relationship with development level, and changes compared with 2006. J Pain Symptom Manage. 2014;47:283-97. 